\title{
Envejecimiento poblacional: discriminación y políticas públicas integrales
}

Ageing population: discrimination and integral public politics

Óscar Osorio Pérez*

Universidad Nacional Autónoma de México, Ciudad de México, México DoI: http://dx.doi.org/10.28928/revistaiztapalapa/812016/atc6/osorioperezo

\section{Resumen}

Las experiencias del proceso natural de envejecimiento se reproducen en contextos de inseguridad social que hacen de los ancianos personas extremadamente vulnerables a la discriminación; sin embargo, sugiero que no son los prejuicios y estereotipos, en primera instancia, causantes de aquella, sino las condiciones de miseria y desigualdad en las que viven. Puesto que las condiciones de vida de los ancianos son un reflejo de las condiciones de vida de la población más joven, se requiere implementar políticas públicas de atención integral; de lo contrario, la población adulta estará condenada a padecer eternamente los problemas que hoy enfrentan.

Palabras clave: envejecimiento demográfico, estereotipos, políticas públicas.

\begin{abstract}
Experiences learned from the natural aging process are reproduced in a context of economic and social insecurity, which makes extremely elderly people vulnerable to discrimination. Research shows that discrimination against older people is not confined to the socially constructed stereotypes and prejudices towards the elderly, but the source is in conditions of poverty and inequality. Since the living conditions of the elderly are a reflection of the situation of young people, tackling the problem of discrimination need to complement comprehensive public policies that
\end{abstract}

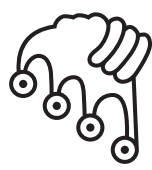

IZTAPALAPA

Agua sobre lajas
* Facultad de Estudios Superiores Zaragoza Campus III oscar.osorio.perez3@ gmail.com 
guarantee social security and access to justice for all people. Otherwise, senior will be eternally condemned to suffer the same problems that actually put face to face.

Keywords: Population ageing, stereotypes, public policies. 


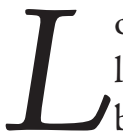

os estudios sobre los procesos de envejecimiento y la vejez han mostrado la necesidad de buscar relaciones sistemáticas entre los procesos de orden biológico -como el envejecimiento del cuerpo y la enfermedad-, con aquellos de carácter social -como las trayectorias de vida laboral o familiar, la pertenencia a una clase, etnia y género-, que definen las experiencias de envejecer en los marcos de condiciones estructurales como el desarrollo histórico, condiciones sociales, políticas económicas y la organización institucional del país, que van configurando las trayectorias y condiciones de vida de un sector importante de ancianos en México. ${ }^{1}$ Lo anterior no se adhiere a las propuestas metodológicas estratigráficas que suponen una relación entre los factores biológicos, psicológicos, sociales y culturales, sino a una concepción sintética de las anteriores variables, integrando teorías y conceptos a partir de los cuales "se puedan formular proposiciones significativas que abarquen conclusiones ahora confinadas en los campos de estudio separados" (Geertz, 1997: 46-5I). Con lo anterior sugiero no reducir el análisis de la vejez y el envejecimiento a una perspectiva holística, sino buscar relaciones sistemáticas entre diversos fenómenos. Algunos estudios de la vejez, desde la perspectiva de género, por ejemplo, nos muestran que las mujeres enfrentan más problemas en la vejez que los hombres debido, entre otras razones, a la exclusión de derechos a las que históricamente han estado sujetas (Montes de Oca, 1999; Garay y Montes de Oca, 20II; Rojas, 20I2). Si bien las condiciones de pobreza inciden en las trayectorias de la vejez, los anteriores estudios nos muestran que es el reconocimiento de derechos y garantías de seguridad social lo que, sin determinar, dispone y constituye la calidad de vida de las personas, y en particular la del adulto mayor.

1 El presente documento forma parte de los resultados de una segunda estancia de investigación posdoctoral realizada en el Centro de Investigaciones y Estudios Superiores en Antropología Social Unidad Golfo, durante 2014. Agradezco al Conacyt el financiamiento de la investigación y a los doctores Felipe R. Vázquez Palacios y Saúl Horacio Moreno Andrade por los apoyos y orientación brindados durante mi estancia. Agradezco también la valiosa colaboración de Karol Aponte del Ángel en el proceso de investigación. 
Los estudios de las condiciones de vida de los ancianos en México, y en buena media en Latinoamérica, apuntan generalmente al análisis de la situación de miseria en que vive buena parte de la población adulta. Lo anterior se debe, quizás, a que hay en las ciencias sociales una arraigada tradición de investigar temáticas relacionadas con problemas que enfrentan las sociedades actuales, tal vez con la esperanza de contribuir a su entendimiento, generar propuestas y posibles vías de solución. No es casual que la mayoría de estudios sobre la vejez nos hablen de la pobreza, desigualdad, marginación y un mundo de experiencias adversas a las que se enfrenta este sector de la población. El problema se complica cuando observamos que en México los adultos mayores conforman uno de los sectores más desfavorecidos de la población, muy a pesar de la existencia de programas y políticas públicas orientados a mejorar sus condiciones de vida. Experiencias derivadas del proceso natural de envejecimiento, como lo son problemas de salud y disminución de capacidades físicas, se reproducen en un contexto de inseguridad económica y social, que hace probable la expresión de conductas discriminatorias hacia los adultos mayores y reduce las posibilidades de acceso a una vida digna, haciendo mucho más inestable la experiencia de envejecer. Ante esta situación, una vertiente importante de estudios de la vejez y prácticas discriminatorias se ha mantenido anclada en por lo menos dos problemas epistemológicos que obstaculizan su conocimiento y la consecuente propuesta de políticas públicas eficientes: proposiciones deterministas y sobredimensionadas con las que describen situaciones adversas a las que se enfrentan los adultos mayores, como es la discriminación; y la atomización excesiva de derechos con los que se pretende combatir problemáticas específicas de este sector poblacional, sin considerar otras generales que afectan al conjunto total de la población.

El objetivo de la investigación es mostrar que el problema de la discriminación hacia las personas mayores es un problema que se desprende de otros de carácter estructural, como son las condiciones de miseria y desigualdad que buena parte de este sector y la población en general padecen; de modo que atender la discriminación hacia la vejez implica necesariamente mejorar las condiciones de vida de toda la población. Resulta necesario, entonces, implementar políticas públicas orientadas a combatir la discriminación hacia la vejez, situando a las personas mayores como integradas a una sociedad que enfrenta problemáticas similares a las suyas, de tal manera que si no se atienden las demandas de la población en general, los adultos mayores, como sector poblacional, estarán condenados a padecer eternamente las mismas dificultades que hoy enfrentan. 


\section{Diseño de la investigación}

Durante el proceso de investigación me he preguntado acerca del impacto que tiene la discriminación en la vida cotidiana de los adultos mayores; de sus causas y posibles vías de atención, de su prevención y sus efectos. Me pregunto también acerca del alcance e impacto de los prejuicios y estereotipos socialmente construidos, y si estos están dirigidos efectivamente al menosprecio de la vejez, como proceso biológico, y a las personas mayores en general. He reflexionado sobre el impacto que han tenido las políticas públicas dirigidas hacia la vejez cuyo objetivo, se supone, ha sido el de mejorar sus condiciones de vida, $y$ he concluido que es necesario superar las barreras del asistencialismo mediante posibles caminos que orienten políticas públicas desde una perspectiva integral, que no solo se ocupen de las problemáticas que enfrentan los ancianos en nuestros días, sino que atiendan las necesidades de la población más joven, de modo que sean también un asunto de prevención, y extensivas a otros sectores sociales. Algunas de las preguntas que guían la presente investigación surgen de la necesidad de comprender, entre otros problemas, ¿cuál es la relación entre prejuicios y estereotipos con las conductas discriminatorias? ¿Qué impacto tiene la discriminación dirigida a personas en condiciones de miseria y desigualdad? ¿Es la vejez por sí misma blanco de discriminación? ¿Qué condiciones de vida hacen de los ancianos personas vulnerables a la discriminación? ¿Es posible combatir las conductas discriminatorias hacia los adultos mayores? ¿Cómo pueden las políticas públicas prevenir y enfrentar el problema de la discriminación hacia las personas mayores? ¿Deben las políticas públicas construirse a partir de necesidades específicas de cada uno de los sectores de la población? ¿Es posible construir políticas públicas de prevención y no solo de atención hacia la vejez? ¿Qué ventajas puede ofrecer un modelo de políticas públicas que, al mismo tiempo que atienda las demandas de la población mayor, contemple e incluya las demandas de las personas más jóvenes, ocupándose de la prevención tanto como de la atención? Mi intención no es resolver las anteriores preguntas de manera definitiva, sino construir vías de análisis y propuestas que contribuyan a pensar cómo mejorar la calidad de vida de las personas.

Como aportes de la investigación pretendo mostrar que el desinterés por garantizar el acceso a los mecanismos de justicia y seguridad social permite que la población en general, y en particular los ancianos, sean objeto de discriminación y violencia. Con lo anterior sugiero que son las condiciones de desigualdad las que hacen de los ancianos un sector vulnerable a la discriminación, por lo que considero erróneo afirmar que los ancianos padecen una discriminación sistemática a causa de los prejuicios y estereotipos socialmente construidos en torno a la vejez. En todo caso, los 
prejuicios y estereotipos se detonan en contextos donde las condiciones de vida de las personas en general, y de los ancianos en particular, tienden a ser desfavorables, haciéndolos extremadamente vulnerables a padecer actos de discriminación y, en consecuencia, al no reconocimiento de sus derechos fundamentales. Intento mostrar también que las políticas púbicas dirigidas a la vejez y al envejecimiento de la población en México son incapaces de combatir problemas sustanciales a los que se enfrentan los ancianos en el trayecto de su vida cotidiana, porque son esencialmente políticas diseñadas desde una óptica asistencialista y carecen de la franca intención de contribuir a mejorar su calidad de vida. Por lo anterior, considero necesario el cambio de paradigma que sitúa a los ancianos, y a otros grupos vulnerables, como sujetos de protección y socorro a favor de quienes deben implementarse políticas públicas de carácter asistencialista. En su lugar, sugiero que las políticas públicas deben garantizar el acceso a los mecanismos de impartición de justicia y a la seguridad social, considerando a los ancianos como sujetos de derechos y no solo como sujetos de asistencia. Lo anterior exige incorporar en las políticas públicas dirigidas exclusivamente a atender las necesidades de la población mayor una perspectiva integral que permita la identificación de áreas de intervención particulares, y al mismo tiempo contemplen las necesidades de la población más joven, de manera tal que se garantice una vejez autosuficiente y estable a partir de políticas de prevención y no solo de atención.

\section{El contexto de las políticas púbicas de la vejez en México}

Una política púbica es uno de los mecanismos de intervención que usa el Estado para favorecer las condiciones de vida de la población en general. Toda política pública tiene como punto de arranque la elección de un problema que es necesario atender, a través de una agenda que defina su diseño, desarrollo, implementación y evaluación (Henríquez, Johannsen y Morales, 2015: 135-146). La función esencial de una política pública es encontrar soluciones a problemas de interés público. Como competencia de gobierno, "incluye todo aquello que estos deciden hacer o no hacer, debido a que el no actuar es un actuar del poder público y puede tener el mismo impacto sobre la sociedad" (Thomas Dyle, citado en Fernández, 1997ः 464). Como toda acción gubernamental, las políticas públicas no están exentas del halo mítico que recubre sus funciones. Su expresión utópica supone que estas: 
[... ] recogen las demandas de los sectores civiles y políticos, las reelaboran en objetivos y acciones legalmente compatibles, aprovechan la información y el conocimiento que existe en una sociedad para seleccionar las acciones y las formas de organización capaces de producir los objetivos decididos, e incluyen el cálculo de los recursos públicos que las acciones necesitan para operar y alcanzar los resultados esperados (Aguilar Astorga, citado en Razo, 2014: 80).

Pero aunque las políticas públicas son esencialmente programas de acción gubernamental o de una autoridad pública, con normalidad surgen en colaboración con organizaciones e instituciones civiles y de la iniciativa privada, razón por la cual también "están sujetas y mediadas por intereses políticos y económicos distintos al interés público, donde las limitantes financieras, de personal y planificación, son determinantes" (Salazar, 2015: x). Es necesario también considerar que toda política púbica es el resultado de la acción u omisión de élites políticas y su relación con la estructura del sistema económico, puesto que, como lo señalan Meny y Thoenig (1992: 267) "Una política pública es, a la vez, una decisión política, un programa de acción, una movilización de actores y de instituciones para la consecución de objetivos más o menos definidos". No intento con lo anterior reducir el proceso de producción de políticas públicas a concesiones de poder y dominio mal intencionado, aunque el mundo de las políticas públicas se sujete también a los juegos de una jerarquía política: inoperatividad burocrática, desvío de recursos, distorsión de objetivos y resistencia del control administrativo, entre otros obstáculos (Fernández, 1997 : 475-479), tal como sucede en América Latina donde las políticas "todavía tienden a canalizar beneficios privados hacia ciertos individuos, facciones o regiones, y no a favor del interés público" (Scartascini, 2015: 23). Por lo anterior, al poner en consideración la viabilidad y el impacto de una política pública no debemos menos que considerar la arena donde se debate, propone y argumenta, a partir no solo de consideraciones de interés público, sino también de juicios morales, aspectos emotivos, creencias e intereses particulares.

Para Sandra Huenchuan $(2003 \div 2)$ los componentes ideales de una política de vejez son tres: una base institucional, un sustento legal e intervenciones concretas como los planes, programas y proyectos. En México, "la base institucional es el INAPAM; el sustento legal está en la Ley de los Derechos de las Personas Adultas Mayores, y las intervenciones concretas corresponden al Acuerdo Nacional a Favor de las Personas Adultas Mayores" (Muñoz, 20II*38). Al igual que en varios países latinoamericanos donde el envejecimiento poblacional tiene consecuencias directas en los sistemas de protección social (Jaspers, 2007bः 9-Io), en México se han 
sugerido programas de asistencia en dos grandes campos: en el de la salud, debido a la enorme demanda de un acceso universal y de calidad en la atención sanitaria,y en lo que respecta a la seguridad social, donde se reclama el aumento de la cobertura y calidad de las pensiones y jubilaciones. Sin embargo, desde la creación del Instituto Nacional de la Senectud (INSEN), en 1979, hasta ahora, las políticas públicas dirigidas al adulto mayor se resumen en programas de atención que no han resuelto los problemas básicos de seguridad social y salud, pero que han sido utilizados como programas asistencialistas y de política de promoción partidista y coacción del voto (Razo, 20I4). En este sentido, los ancianos se han convertido en un sector social en disputa por la clase política. El aumento de la población senescente y las condiciones precarias y de desventaja en la que viven buena parte de ellos los convierte en fuente potencial de votos, siempre que se dirijan políticas asistenciales oportunistas. Lo anterior sugiere que en México no hay políticas públicas para los adultos mayores, sino estrategias asistencialistas (Rosas, 2013). Las políticas públicas no deben buscar resolver la vida a los ancianos, ni crear derechos o políticas de privilegio, sino establecer condiciones de bienestar y seguridad social que posibiliten una vejez plena.

\section{La vejez: estigmas y estereotipos}

En aras de mostrar que la vejez es una construcción histórica, social y cultural y que, por lo tanto, los problemas que afectan la vida de los ancianos hoy pueden resolverse a partir de la concientización social y el compromiso político, se ha creado toda una mitología que coloca al anciano en la cúspide de la estratificación social de las sociedades antiguas y tradicionales (Piña, 2006; Lozano, 2009), sustentada en un arsenal de fuentes históricas, religiosas, artísticas, literarias y científicas, que han contribuido a crear una imagen idílica del anciano en otros momentos históricos (Polo y Martínez, 2oro; Reyes et al., 2013).

En realidad, el conocimiento del estatus, los roles y los tratos que se otorgaban a la vejez en las sociedades antiguas resulta un tanto especulativo debido a la escasez de fuentes históricas que hagan referencia directa al tema. Lo que sí es un hecho es que la historia de la vejez tiene un horizonte histórico y cultural propio, presentándose variopinta y harto paradójica. Por supuesto, el análisis histórico de la vejez ha mostrado la presencia de ciertas representaciones hegemónicas y dominantes en diferentes periodos históricos (Alba, 1992; Rodríguez, 1989). Aun así, la vejez presenta sus formas problemáticas tanto en las sociedades occidentales modernas e industrializadas, como en casi todas las sociedades conocidas, incluidas las 
sociedades cazadoras-recolectoras y las aldeanas; las antiguas y modernas con sus particulares modos de producción. La historia de la vejez nos muestra también que no es la vejez en sí misma, sino la marca del paso del tiempo en objetos y personas en un contexto crítico, lo que genera concepciones y significados que derivan en una posición particular sobre la vejez. El envejecimiento se asocia inevitablemente con la muerte-pérdida y, en consecuencia, con el infortunio; refleja el paso del tiempo que deja su marca indeleble, como una promesa del fin. La tensión que genera el paso del tiempo y las amenazas de la naturaleza, que impactan psíquicamente, se han expresado en actos rituales de sacrificio con los que se enfrentan las amenazas y que al mismo tiempo permiten generar cohesión social. Desde las sociedades recolectoras hasta aquellas organizadas en Estados-nación, los ancianos, junto a otros sectores de la población, han sido objeto de sacrificio -chivos expiatorios por excelencia-en momentos de alta tensión y eminente peligro: hambrunas, catástrofes, guerras. Tanto en las sociedades tradicionales como en las modernas, los acianos mantienen cierto estatus cuando las condiciones de vida, en general, son buenas, pero este se degrada en momentos de alta tensión y circunstancias adversas. Por eso, las condiciones de vida de los ancianos son un reflejo de los avatares de las de la vida cotidiana, y una respuesta a las aspiraciones de reproducción social en el orden material y simbólico.

En ocasiones, al hablar sobre problemáticas que enfrentan ciertos sectores sociales considerados de facto vulnerables pueden hacerse generalizaciones explícitas que intentan caracterizarlos, pero que van más allá de expresiones descriptivas y terminan por constituirse en estigmas: señas de identificación que parten de representaciones habituales como referencias codificadas y abreviaturas culturales. El envejecimiento, como proceso biológico y cultural, implica la inevitable transformación de la persona, tanto en lo físico como en lo social. Como categoría conceptual no puede precisarse sin el riesgo de obviar o desdeñar alguno de sus atributos, estatizando unos y relativizando otros; de aquí que sus concepciones aparezcan, en ocasiones, tan ambiguas e inciertas. Algunas investigaciones, que tratan de explicar la discriminación de la que son objeto las personas mayores, suponen que son los estereotipos negativos asociados con la enfermedad, la fragilidad y la decadencia, junto con los aspectos físicos en deterioro como la aparición de canas, arrugas y postura encorvada, la causa del trato discriminatorio que padecen (Sánchez, 2004: 29; Lasagni et al., 20I2: 3). Según este punto de vista, las consecuencias de tal percepción redundarían inevitablemente en la exclusión de derechos y oportunidades fundamentales para el acceso a una vida digna y satisfactoria independiente de la edad (Moreno, 20II: 2-5; INAPAM-CONAPRED, 2OI2: 9). Lo anterior sugiere que de los estereotipos de la vejez -percepciones erróneas asociadas con la enfermedad, el deterioro mental, 
la reducción de capacidades físicas y otras habilidades- "se desprenden actitudes negativas que están en la base para la marginación por motivos de edad" (Sánchez, 2004: 2). Sin embargo, tal como lo señala Montes de Oca (2013: 26),"en gran parte de la literatura sobre vejez no se precisa el impacto de prejuicios, estereotipos y estigmas hacia la población adulta mayor".

En mi opinión, el señalamiento del deterioro de la salud y de ciertas capacidades físicas no forma parte de un prejuicio hacia la vejez; en todo caso, es la observación de un hecho natural del proceso de envejecimiento, como la aparición de arrugas y pelo cano. El problema de fondo radica en que, para un sector importante de ancianos, la experiencia de envejecer se encuentra cara a cara con la experiencia de vida en un contexto adverso, done las posibilidades de una vejez autosustentable puede llegar a ser inasequible, incluso para quienes tienen algún tipo de seguridad social. Esto se debe, entre otras razones, observa Ham (1999: I0) a que la vejez:

[...] produce un regreso a la dependencia hacia la familia en particular, y hacia la sociedad en general, con sustanciales demandas de manutención y cuidado, puesto que se incrementan los riesgos de pérdida de las capacidades físicas y mentales, disminución de la autonomía y la adaptabilidad, menoscabo de roles familiares y sociales, retiro del trabajo, pérdida de capacidad económica, cese de otras actividades y deterioros en la salud de consecuencias incurables y progresivas.

De ahí que la vejez se traduzca en un proceso inestable y de riesgo, que coloca a los ancianos en situaciones de vulnerabilidad frente a condiciones adversas del mercado de trabajo, cobertura de seguridad social deficiente e inseguridad económica crítica (Huenchuan y Guzmán, 2006).

En oposición a lo que se considera una visión estereotipada de la vejez, en ocasiones suele resaltarse de ella atributos tales como experiencia, sabiduría y paciencia; en tanto que elementos como deterioro y muerte suelen ocultarse, valorándose así atributos positivos del envejecimiento y negándose aquellos que, se supone, degradan la condición de las personas mayores (Lyonnais, 1965; Moragas, 1998). Sin embargo, negar las consecuencias del envejecimiento, por considerarlas prejuiciosas, equivale a estereotipar la vejez misma, de manera tal que al proponer estrategias de intervención estas partirán de idealizaciones y no de experiencias propias de la vejez y el envejecimiento. La implicación de las percepciones y concepciones de la vejez, en relación a las políticas públicas, es relevante porque son el punto de partida para la elaboración de políticas públicas orientadas a mejorar las condiciones de vida de los ancianos, especificando áreas de intervención y configurando prioridades. 
Por otra parte, aunque no se han estudiado bien las posibles causas positivas de los estereotipos, es importante mencionar que con frecuencia las personas no elaboran categorías particularizadas de otras personas, sino que, con base en supuestos atributos o características específicas, se construyen modelos estandarizados que se expresan como estereotipos internalizados en campos semánticos, que estructuran interpretaciones y creencias como expectativas acerca de los miembros de un grupo (Brewer, Dull y Lui, 1981: 658). Por lo tanto, los estereotipos no necesariamente detonan conductas discriminatorias y sí pueden postularse como elementos de proximidad al conocimiento de una persona o grupo. Al respecto, Ham (1999: I0) enfatiza en que "Con todo y lo difícil que resulta aceptar los aspectos negativos de las edades avanzadas y los intentos por encontrar ventajas al envejecimiento, finalmente se cae en la necesidad de resolver los distintos problemas que este proceso conlleva, incluyendo su prevención". Así entonces, escribe Huenchuan (2007: 9-IO, I20-I29), en relación a la salud: el envejecimiento de la población demanda nuevas prestaciones y tratamientos, acceso universal y de calidad en la atención sanitaria, promoción y prevención de la salud; en materia de seguridad social requiere financiamiento tanto de los sistemas contributivos basados en el reparto como de los sistemas de pensiones no contributivas y el aumento de la cobertura y la calidad de las pensiones y jubilaciones, fomento del ahorro y promoción de actividades económicas y sociales.

\section{Vejez y discriminación}

Un tópico retórico en los estudios de la vejez es que con el devenir del capitalismo y la industrialización -donde el progreso se mide a partir de la técnica que impulse la producción, genere rentabilidad y permita la acumulación- los ancianos han perdido el lugar que antaño se les confería. La situación crítica que viven los ancianos de hoy se debe, según el tópico, al modelo económico dominante en la modernidad: el capitalismo. Es en el marco del moderno capitalismo donde, al hablar de discriminación, se pone el acento en aquella que padecen ciertos grupos sociales como el de las mujeres y la población adulta mayor, para quienes según Bucio y Lucas (20II: 7) "se presenta de manera histórica, constante y sistemática". En mi opinión, resulta bastante cómodo señalar que la causa de las lamentables condiciones en que viven los adultos mayores, de la discriminación en particular, se debe al modo de producción o al modelo económico llamado capitalista o capitalismo neoliberal, sin mostrar las situaciones y los contextos en que se produce. Contrario a esta posición, considero importante valorar el tipo de discriminación dirigida a los adultos mayores partiendo 
de un conocimiento más apegado a las experiencias que enfrentan en la vida diaria que a los constitutivos conceptuales que surgen de la teoría en bruto, con la finalidad de construir políticas públicas más apropiadas y convenientes.

En un primer momento podemos entender por discriminación la acción encaminada a construir una diferencia: separar y distinguir. En un sentido más sociológico, un acto discriminatorio es la tendencia a expresar un trato diferenciado a algo o a alguien que es considerado con menor valor o inferior. Es importante distinguir entre la discriminación intencional o directa, que excluye o restringe el ejercicio de un derecho, y la discriminación de facto o simbólica, encarnada en el lenguaje, en el universo de sentido y significados de los sistemas culturales, que puede ser intencional o no, pero con consecuencias igualmente restrictivas al goce y ejercicio de derechos.

$\mathrm{Al}$ adherir el elemento sistemático a los actos de discriminación intentamos profundizar en la semántica con la que se intenta enfatizar que el acto discriminatorio no es un acto en solitario y sin sentido, sino que refleja premeditación y un interés particular, que se reproduce y refuerza en los marcos de la vida cotidiana. Remite también a situaciones en que no se requiere el acto discriminatorio directo, pues no necesariamente involucra a actores que inflijan daño, siendo equivalente a la injusticia social, puesto que está embebida en las estructuras sociales (Galtung, 1994). En este sentido, la discriminación sistemática hace referencia también al "trato diferenciado que tiene implicaciones de desventaja cuando produce un daño moral o físico, y obstaculiza la satisfacción de cubrir necesidades básicas como resultado de su conexión con estructuras sociales que reproducen distribuciones inequitativas de poder y recursos" (Weigert, 1999: 432). La discriminación sistemática normalmente se dirige a un sector de individuos indeseables, ante quienes se construyen elementos de discriminación con fines diversos. Tiende a ser mucho más recurrente y efectiva cuando se dirige a quienes Zizek (2009:25) denomina personas desechables: excluidos, sin techo y desempleados, que carecen de los recursos y medios necesarios para enfrentar los actos discriminatorios y su consecuente violencia, como el despojo de sus derechos humanos elementales y la imposibilidad de acceder a la justicia. En lo sucesivo pretendo mostrar que si bien los actos discriminatorios hacia una persona están íntimamente relacionados con su pertenencia a un grupo o sector social, normalmente se superponen otros factores y situaciones que potencian la discriminación, como lo son la enfermedad y la pobreza.

Para algunas personas mayores enfrentar situaciones de discriminación y violencia es una constante. El menoscabo de sus derechos humanos, la inseguridad social, la restricción a los servicios de salud y la falta de empleo tienen como consecuencia 
la degradación personal y la respectiva disminución de su calidad de vida. En este contexto, no es que socialmente los adultos mayores padezcan de discriminación intencional y programada por parte de un sector social en particular o de un poder público, o que se construya un orden de miedo hacia las personas envejecidas, se fomente el odio o desprecio por causas propias de su edad. Lo que sucede es que cuando la vejez se reproduce en contextos de miseria, en condiciones de desigualdad y desventaja, es relativamente fácil que se detonen prejuicios y estereotipos que motivan su desprecio o aversión. Así pues, factores como la pobreza, la enfermedad y el abandono, que padece un sector importante de la población adulta, los convierte en blanco asequible de discriminación.

Un perspectiva que aún suele utilizarse para mostrar y explicar las fuentes de discriminación hacia la personas mayores es el vijeismo, el cual supone que los ancianos son discriminados por motivos propios de la edad y las percepciones negativas derivadas de esta (Hausdorff, Levy, Wei, 1999: 1346). El viejismo puede expresarse en "actitudes como conductas cotidianas discriminatorias y en prácticas institucionales que se relacionan y refuerzan mutuamente, contribuyendo a la transformación de la vejez como proceso natural en un problema social, donde las personas mayores soportan condiciones que les perjudican" (Palmore, I990: 36). Viejismo es un concepto derivado de ageism con el que Butler (1969: 222) se refería al "proceso de estereotipia y discriminación sistemática contra las personas por motivos de la edad, de la misma forma que el racismo y el sexismo se originan por el color de la piel y el género". En nuestros días, se ha extendido el término ageism como una alteración en los sentimientos, creencias o comportamiento en respuesta a la edad cronológica percibida de un individuo o un grupo de personas, que puede operar sin ser advertido, controlado o con intención de dañar de manera consciente (Becca y Banaji, 2004: 51).

Si bien una extensa literatura sobre investigaciones de la vejez afirma la existencia de conductas discriminatorias hacia los ancianos por el solo hecho de serlo, también se han realizado investigaciones donde se muestra que el denominado viejismo, como actitud prejuiciosa y discriminatoria, se ha sobredimensionado. Estrictamente hablando, en los términos en que Butler se refirió al ageism, parece que se ha exagerado al equiparar los estereotipos sobre la vejez, que hacen referencia a elementos de carácter cognitivo, con actitudes discriminatorias y al acto mismo de hacer efectiva la discriminación (Brubaker y Powers, 1976; Schonfield 1982; Cook, 1992; Cohen, 200I). La literatura que habla de discriminación sistemática hacia la vejez y el viejismo no ha mostrado que un sector de la población en especial reproduzca actos discriminatorios continuos en contra de los ancianos. Aunque si 
bien, por ejemplo, Lovell (2005:22) muestra que algunos estudiantes de enfermería tienen una actitud negativa hacia las personas mayores, no se concluye del hecho que tengan por actitud generalizada la discriminación; menos aún que los jóvenes expresen sistemáticamente conductas discriminatorias hacia los ancianos. Lo anterior se debe a que la discriminación no es en sí misma una actitud decretada y concluyente y, dirigida hacia la vejez, está estrechamente relacionada con algunas de las variables sociodemográficas como son, por ejemplo, la ocupación y escolaridad, corroborándose la existencia de diferentes niveles de estereotipos negativos hacia la vejez en varios países de América Latina (Lasagni, 2012).

Investigaciones sobre organización cognitiva revelan que existen múltiples estereotipos o subcategorías hacia las personas mayores, algunos positivos y otros negativos (Chasteen, Schwarz y Park, 2002: 54I), por lo que las actitudes hacia las personas mayores tienden a ser variadas, $y$ aunque efectivamente se expresan estereotipos negativos hacia la vejez, las personas más jóvenes no ven estereotipos negativos como lo más típico hacia los ancianos (Hummert, 1990ः 188). Por ejemplo, los niños normalmente muestran una percepción y actitud muy positiva hacia ellos (Davidovic et al., 2007: 1138), en tanto que los jóvenes muestran generalmente percepciones y actitudes más positivas que negativas hacia la vejez (Axelrod, y Eisdorfer, 196I: 78; Dulcey y Ardilla, 1976: 65-66; Matheson, Collins y Kuehne, 2000: 247). Incluso, las mismas investigaciones muestran que son los adultos mayores quienes construyen estereotipos más complejos hacia sí mismos, y en ocasiones más negativos de los que los más jóvenes hacen de ellos.

No intento con lo anterior negar que las percepciones cognitivas estereotipadas puedan tener como consecuencia actitudes discriminatorias, sino mostrar que existe una enorme diferencia entre ambas acciones. Un acto discriminatorio parte necesariamente de percepciones negativas y estereotipadas, pero las percepciones estereotipadas no necesariamente expresan actitudes discriminatorias. Lo anterior sugiere que las percepciones pueden orientar actitudes, pero no determinarlas. Además, como una característica de los estereotipos hacia la vejez, que difícilmente se reproducen hacia otros grupos sociales, es que inspiran concepciones positivas y negativas al mismo tiempo (Palmore, 1990). Dichas concepciones nunca permanecen fijas, sino que comprenden constelaciones poco estructuradas de rasgos que los perceptores reconocen no siempre exactos de los miembros individuales de una categoría (Matheson, Collins y Kuehne, 2000: 246).

Cuando se afirma que las personas adultas mayores padecen una discriminación sistemática por causas propias de la edad se promueve la inoperante simplificación que se dice combatir. Si se pretende que las personas abandonen las simplificacio- 
nes demasiado generales que tan a menudo se hacen sobre el envejecimiento, se tiene que dejar de hacer preguntas de actitud sobre las personas mayores como si fueran un grupo homogéneo (Cook, 1992: 293). Por ejemplo, aun cuando un número considerable de adultos mayores (27\%) dijo, en la Encuesta Nacional sobre Discriminación en México 20Io, haber padecido alguna vez discriminación por la edad, debemos considerar que la discriminación por edad no necesariamente existe porque algunas personas de edad avanzada pueden creer que sucede, y generalizar la discriminación por edad puede sobrevalorar el hecho (Cohen, 200I: 557) puesto que las respuestas son también un asunto de percepción y creencia. No considerar la singularidad de las fuentes y consecuencias de la discriminación hacia las personas adultas es tanto como afirmar que los pobres son criminales porque un porcentaje de ellos se dedica a delinquir, o que los ricos son filantrópicos porque una parte de ellos realiza obras de caridad.

Una investigación sobre el tipo de violencia que percibe hacia sí mismo el adulto mayor en México encontró que, del total de la población estudiada, $42.3 \%$ se percibió con algún tipo de violencia intrafamiliar psicológica, seguida por abandono o negligencia (Vargas, et. al., 20II: 65). En 2014, la Fundación para el Bienestar del Adulto Mayor reportó que de los I0.9 millones de adultos mayores que hay en México, I.7 millones son víctimas de violencia tanto física como psicológica, y $40 \%$ vive esta realidad dentro de sus propios hogares (De Regil, 2014). Efectivamente, en los marcos de la vida cotidiana personas adultas mayores sufren discriminación, incluso pueden padecer experiencias de violencia continua y prolongada. Pero de lo anterior es importante precisar que los ancianos no padecen discriminación y violencia solo a causa de su edad, sino"por su condición de fragilidad, vulnerabilidad a ciertos riesgos, falta de autonomía o de capacidad, que los convierte en personas que pueden ser fácilmente objeto de abuso o maltrato" (Jiménez, 2012: 24).

La Encuesta Nacional sobre Discriminación en México 2010, en lo referente a las personas adultas mayores, muestra que ante la pregunta de si alguna vez ha sentido que sus derechos no han sido respetados por su edad, 24.1 \% respondió que sí, mientras que $70.9 \%$ respondió que no. En tanto que de las mismas personas encuestadas, $36 \%$ refieren que los principales problemas a los que se enfrentan por cuestiones de su edad son los de carácter laboral, seguidos de los problemas de salud y discapacidad ( $15 \%$ ). Solo 9.9\% considera la discriminación, la intolerancia o el maltrato como su principal problema. Por supuesto, el hecho de que poco más de $24 \%$ de los adultos mayores considere haber sufrido algún tipo de discriminación es un indicador de alerta. Pero al situarse en la discriminación en sí misma se corre el riesgo de soslayar factores como las condiciones de desventaja y desamparo en que 
viven. La misma encuesta revela que las tres dificultades más importantes a las que se enfrentan los adultos mayores son: las de carácter económico, 40.3\%; de salud: acceso a servicios sanitarios y de suministro de medicamentos, $37.3 \%$; y laborales, $25.9 \%$. Por lo anterior parece, entonces, que el problema no es la discriminación en bruto, sino la falta de empleo e ingresos que les permitan autonomía económica, acceso a servicios sanitarios eficientes, falta de seguridad social y derechos, problemas de los cuales se desprende el de la discriminación. Lo que se presenta es una actitud discriminatoria que surge de la marca que imprime la pobreza, del rechazo a la miseria, la falta de acceso a la justicia y el desinterés por que las cosas funcionen mejor.

\section{Problemas adyacentes al envejecimiento demográfico}

El proceso de transición demográfica que experimenta América Latina, donde la población de edad avanzada crece a ritmos mucho más acelerados que la de otros grupos de edad, requiere con urgencia reorientar políticas públicas más justas e incluyentes, que permitan atender no solo las necesidades de los adultos mayores de hoy, sino también las de los jóvenes de hoy que serán adultos mayores mañana. La demanda surge no solo por la necesidad imperiosa de mejorar las condiciones a las que se enfrenta la población mayor en el proceso de satisfacer sus necesidades y elevar su calidad de vida, sino también por los problemas adyacentes y aquellos que se desprenden de políticas públicas deficientes. Las causas residuales de un paulatino envejecimiento demográfico comienzan a generar competencia por recursos sociales para la atención entre los distintos grupos de edad, así como el aumento del empobrecimiento y la desigualdad entre los viejos (Robles et al., 2006). Por lo que para modificar el estado de cosas es necesario partir de tres ejes: "solidaridad, justicia y equidad, con el fin de universalizar los beneficios de la seguridad social a toda la población" (Alonso, 20I2: 37).

De acuerdo con cifras del Consejo Nacional de Población (ConApo) en México hay II.7 millones de personas mayores de 60 años ( $15 \%$ de la población total a partir de 2015). De estas, 31.5\% están en una etapa de prevejez (60 a 64 años); 41.I \% se encuentran en una vejez funcional (65 a 74 años); I2.3 \% en vejez plena ( 75 a 79 años) y $15.1 \%$ transita por una vejez avanzada ( 80 años y más). A pesar de las actividades económicas que desempeñan, se estima que 6I \% reciben algún tipo de apoyo y seguridad por parte de su familia, pues más de $75 \%$ de ellos carece de pensiones (SAGARPa, 20I4; CONAPO, 2OII). Como en buena parte de los países en desarrollo, observan Garay y Montes de Oca (20I1: 17), en México los niveles de participación 
económica de los adultos mayores son altos cuando las instituciones de seguridad social advierten una leve cobertura en materia de pensiones, pues la baja cobertura de seguridad social y los reducidos montos de las jubilaciones propician que algunos adultos mayores estén obligados a seguir trabajando. En consecuencia, continúan las autoras, lo que hace a esta población más proclive a la explotación en el mercado de trabajo es su pobreza, que se extiende a los últimos años de su vida.

El cambio demográfico, que apunta a un envejecimiento de la población, hace necesaria la aplicación de políticas públicas diseñadas para atender las necesidades actuales y aquellas que se presentarán a mediano plazo. En consecuencia, no basta con atender las condiciones de la población adulta mayor, sino las consecuencias derivadas del cambio sociodemográfico en todas las dimensiones de la vida: en lo económico, la salud, la seguridad, etc., y hacia todos los sectores poblacionales. Uno de los problemas de la interpretación de los datos anteriormente señalados, que conciernen a las condiciones de vida de los adultos mayores en México, es que pueden leerse de múltiples formas y remitir a conclusiones muy distintas, y en ocasiones arbitrarias. Por ejemplo, CONAPo (2006:29) en sus Proyecciones de la población en México 2005-2050, afirma que el 2020 será su mejor momento:

Dentro de la variada gama de ámbitos en los que inciden los cambios en la dinámica y estructura poblacional de largo plazo, se encuentra el llamado bono o dividendo demográfico. Este concepto se refiere a los eventuales beneficios derivados de una ventana de oportunidad que, por primera y única vez, permanecerá transitoriamente abierta durante las próximas décadas, cuando ocurrirán las condiciones demográficas más propicias para el desarrollo socioeconómico.

Puesto que la mayoría de la población sería joven a partir del 20I2, según CONAPO, estará en edad de trabajar y en adelante la población dependiente será de entre $50 \%$ y $60 \%$. Para 2020 la edad promedio de los mexicanos será de 33 años, momento en que habrá menos niños y adultos que mantener. Pero hasta ahora las proyecciones no se han cumplido y al parecer no serán una realidad puesto que en aquellos cálculos no se contemplaron actitudes, contextos ni problemas adyacentes que impactan directamente en la economía, tales como la injusticia, la inseguridad, la violencia, la corrupción, la migración y la ingobernabilidad.

Otras estimaciones y proyecciones no son tan optimistas y consideran que México no experimenta, sino que padece lo que Rebeca Wong (2006: 26) ha denominado envejecimiento prematuro, dado que la población se desarrolla antes de que lo haga el país. Así, el cambio demográfico que viene acompañado de desempleo, 
demanda de vivienda y de efectividad de los sistemas sanitarios, por ejemplo, ha sido ignorado, por lo que los mismos problemas que enfrentan los adultos de hoy los enfrentarán los adultos del mañana: pobreza, desempleo, falta de seguridad social, integración y derechos. Tal como lo observa Ordorica (2010: 50):

La transición demográfica ha sido muy acelerada en nuestro país: en pocos años tuvimos una población predominantemente joven y, también, en pocos años la población será predominantemente vieja. Primero, no tuvimos el suficiente tiempo para programar las demandas de una población joven y hoy queda ya poco tiempo para atender las demandas de una población en acelerado proceso de envejecimiento. El futuro siempre nos alcanza.

En síntesis, el cambio demográfico que apunta a un vertiginoso envejecimiento de la población mexicana se encuentra de frente a múltiples problemas que no solo no se han resuelto, sino que se han intensificado: la desigualdad y la inseguridad social, la pobreza, la injusticia y la falta de servicios de salud óptimos. Las desastrosas condiciones estructurales en las que se enmarca el cambio demográfico están estrechamente vinculadas a la reproducción de condiciones de marginación y exclusión de un sector importante de adultos mayores en México. Por lo que no basta con la pretensión de combatir el estigma, los prejuicios y estereotipos hacia la población adulta, sino buscar mejorar las condiciones de vida de toda la población, de manera tal que el cambio demográfico deje de exponerse como el "chivo expiatorio" de las causas del subdesarrollo y se atiendan las condiciones estructurales que producen condiciones sociales de miseria (Díaz, 20II).

\section{Orientación de las políticas públicas hacia el envejecimiento demográfico}

Algunas investigaciones sobre la vejez orientadas al desarrollo de políticas públicas parten del supuesto de que estas deben ocuparse de las necesidades concretas de las personas mayores, como son: la pobreza, la inseguridad social y el difícil acceso a fuentes de empleo (Ramírez y Cruz, 2010ः 89). A estas investigaciones se suman los diagnósticos sobre la población adulta mayor en México que encuentran que los problemas de la vejez están ligados a la insuficiencia de pensiones, a un limitado acceso a los servicios de salud de calidad y a la escasa cultura de prevención de enfermedades, por lo que suponen la necesidad de que el Estado tome medidas 
para su protección, tal como lo hace explícito la Secretaría de Desarrollo Social en México (Sedesol, 2013: IO); que, además, añade:

Cuando las personas adultas mayores se encuentran fuera de los esquemas de protección conformados por los sistemas de pensión y jubilación contributivas que ofrecen distintas instancias públicas y privadas, la instrumentación de programas públicos que otorguen pensiones no contributivas se convierte en una medida pertinente de política pública de desarrollo social (SEDESOL, 2013:52).

La anterior perspectiva proteccionista no es muy diferente de la intervención paternalista que el Estado mexicano ha mantenido como política pública-cuyos resultados se reflejan en las condiciones de pobreza de la población en general- que muy a pesar de los saldos "positivos" no funcionan por sí solas, sino que han requerido, en mayor o menor medida, la capacidad organizativa de las unidades domésticas que suministran los apoyos de los programas gubernamentales, cuyas prácticas $y$ mecanismos para enfrentar y sobrellevar la vida en pobreza y los problemas de la escasez de recursos son valorados como los "escenarios de la sobrevivencia" donde operan las políticas asistenciales (González de la Rocha, 2006: 29). Lo anterior refleja uno de los problemas fundamentales en la aplicación de políticas públicas en México: su reducción a una política subsidiaria, en lugar de optimizar los servicios básicos de seguridad social y acceso a la justicia. Es el caso, por ejemplo, de la Secretaría del Desarrollo Social, cuya intervención institucional mediante apoyos y subsidios se supone necesaria para modificar la situación actual que enfrentan las personas adultas mayores e impactar en sus condiciones de vida de manera favorable (SEDESOL, 2013:53).

No sobra decir que el marco de los programas asistencialistas ha sido la corrupción orientada al beneficio de funcionarios públicos y a la coacción del voto. Por lo que no es casual que investigaciones y diagnósticos gubernamentales recurran a proponer soluciones sustentadas en la demanda de recursos económicos. El problema es que las investigaciones de orden académico elaboren propuestas que reproduzcan la postura paternalista y proteccionista institucional. Sin duda, la seguridad económica es necesaria en un contexto donde tres cuartas partes de la población adulta mayor carece de pensiones y más de la mitad requiere para su subsistencia algún tipo de apoyo de su familia; pero la efectividad de las políticas asistencialistas es nula ante la exclusión del entorno de vida habitual y la pérdida de autonomía ante la carencia de seguridad social. Además, si los programas asistencialistas han sido histórica y sistemáticamente portales de corrupción y lucro, sin el funcionamiento 
óptimo de la estructura gubernamental no habrá recursos que alcancen para combatir las condiciones de miseria que buena parte de la población padece. Es el caso, por ejemplo, del programa Sin hambre, al que la Auditoría Superior de la Federación detectó un daño al erario por 147 millones 715 pesos, además del desvío de recursos, privilegio a empresas durante procesos de licitación y pago de servicios no realizados. El hecho representa también la incapacidad del funcionamiento del aparato del Estado, pues no solo se trata de una mala administración de recursos y corruptelas, sino de un pésimo funcionamiento y un conjunto de omisiones propias del Estado para supervisar, dar seguimiento y vigilar la prestación de los servicios convenidos (Tourliere, 2015).

Los enfoques y perspectivas dirigidos al desarrollo de programas de intervención ante problemáticas específicas que enfrentan los ancianos en México han sugerido también proyectos en el orden legislativo y de políticas públicas, que en su mayoría han sobrevalorado la atención a lo que se supone son problemáticas propias de este sector poblacional. Lo anterior significa que con el afán de atender problemáticas particulares de los adultos mayores se presentan propuestas que promueven políticas públicas segregacionistas. Tal cosa ocurre cuando se propone definir políticas públicas para las personas mayores de 60 años, específicas para los de 60 a 70, de 70 a 75 , de 75 a 80 , y para los mayores de 80 años (Caro, 2003: 83). Esta postura imposibilita, de entrada, pensar la investigación y el diseño de políticas públicas dirigidas a las personas adultas mayores considerando su relación con otros sectores de la población, su condición de etnia, género, condición social y nivel educativo, por ejemplo. Puesto que las condiciones de vida de los adultos mayores son un reflejo de la situación que enfrentan sectores más jóvenes, y muchas de las desventajas y condiciones adversas consideradas propias de los adultos mayores las padecen también otros grupos de la población, las propuestas de políticas públicas que se derivan de una visión excesivamente particularista de la vejez obstaculizan el combate de problemas estructurales que perpetúan las condiciones de pobreza, estados de marginación, exclusión y violencia.

\section{Perspectiva integral en la construcción de políticas públicas ante el envejecimiento demográfico}

Enfoques integrales de políticas públicas dirigidas a los adultos mayores se han centrado en cuatro grandes dimensiones: pobreza, integración social, inclusión 
social y derechos, incorporando las perspectivas de clase, género, etnia y salud. A la par, se han construido modelos de asistencia vinculados a la necesidad de legislar particularmente respecto de los derechos de los ancianos a la inclusión social, laboral, de salud y de justicia (Huenchuan, 2009; Marín, 2006; Ruiz, Sánchez y Sanabria 2013; Ribeiro y Mancinas, 2010; Montes de Oca, 2013b).

Huenchaun y Paredes (2006: 66), por ejemplo, muestran que atender como prioridad la seguridad económica de los adultos mayores puede ser conveniente $y$, sin embargo, poco viable, puesto que su viabilidad de momento implica cierto desequilibrio en otras áreas o grupos actualmente considerados como prioritarios:

Los temas de seguridad económica involucran de hecho y solo parcialmente al sistema de seguridad social en sí mismo. La percepción acerca de la prioridad del dinero y la salud hace que poco se visualicen las dimensiones que pueden llegar a adquirir los entornos para el bienestar en la vejez. Esto refiere tal vez a niveles más psicológicos, relacionados con la autoestima y la capacidad de autovalidez, así como con una mayor participación social que haga desprenderse a los viejos de su propio estereotipo.

Con normalidad, la edad suele verse como un factor de vulnerabilidad, ya sea para caer en la pobreza, para ser víctima de marginación y aislamiento social o para no hacer respetar los derechos de las personas adultas (Huenchuan, 2003:13). Pero es necesario apuntar que los anteriores problemas no son propios de un sector poblacional en particular. Por eso, enfrentar el rezago en la calidad de vida de los adultos mayores implica que se considere, al mismo tiempo, la situación de vulnerabilidad en que se encuentra la población más joven en edad de educarse y trabajar, puesto que las situaciones de vulnerabilidad que enfrentan los adultos mayores no son una propiedad de la vejez, sino la consecuencia de desigualdades acumuladas y exclusión de derechos. Por esta razón, la Comisión Económica para América Latina y el Caribe (CEPAL) ha reconocido la importancia de ampliar la cobertura y el perfeccionamiento de la calidad de los sistemas de protección social, para enfrentar los riesgos derivados de la enfermedad, la vejez, la invalidez y la muerte. Por consiguiente, la titularidad de derechos sociales encarna la efectiva pertenencia a la sociedad, pues implica que todos los ciudadanos están incluidos en la dinámica de desarrollo y pueden disfrutar del bienestar que propicia (CEPAL, 2007).

Aunque con poca frecuencia, ante el envejecimiento demográfico se han presentado propuestas de intervención y políticas públicas que consideran como prioridad no solo mejorar la calidad de vida de los ancianos, sino enfrentar los desafíos de los recientes cambios demográficos, cuya tendencia se aproxima cada vez más a un 
envejecimiento de la población. Paz (20I0), por ejemplo, analiza los desafíos que enfrentan los mercados de trabajo y los sistemas de seguridad social en América Latina y el Caribe ante el proceso de transición demográfica y el impacto que tienen las políticas públicas sobre los niveles de pobreza. Su investigación concluye que los ingresos de jubilación, pensión y el trabajo remunerado, son las principales fuentes de sustento de los adultos mayores y, en consecuencia, puerta de entrada a niveles más adecuados de nutrición y salud. Por lo anterior, el desafío no radica tanto en garantizar recursos económicos a los ancianos, sino en el costo y el financiamiento de la seguridad social de la población en general, que incluya opciones de trabajo decente, en el que importa no solo la remuneración económica, sino la libertad, la igualdad, la seguridad y la dignidad.

Al respecto, Sandra Huenchuan (2009) afirma que aun cuando buena parte de la población adulta carece de acceso a prestaciones de seguridad social, servicios sanitarios o servicios básicos, sus condiciones de vida desfavorables están íntimamente relacionadas con un contexto en el que prevalecen altos índices de pobreza, una persistente desigualdad, escaso desarrollo institucional, baja cobertura y calidad de los sistemas de protección social. Por lo tanto, la vulnerabilidad en la vejez no radica propiamente en la edad, sino en la precariedad de los dispositivos de protección establecidos por los Estados, como expresión de una abierta desigualdad y escasa consideración de mejorar las condiciones de vida de este sector poblacional y cubrir las necesidades de las personas en general como un asunto de política pública. Esta perspectiva difiere de las aquellas cuyo discurso monocromático obvian problemas coyunturales y desconocen la posibilidad de crear políticas de intervención para atender los requerimientos del proceso de envejecimiento demográfico; y es de significativa relevancia porque considera también la creación de oportunidades para la población más joven, atendiendo asuntos básicos relacionados con la convivencia y solidaridad entre generaciones, puesto que lo esencial es determinar cómo avanzar hacia la construcción de sociedades más cohesionadas, democráticas e incluyentes.

En relación con lo anterior, Huenchaun y Paredes (2006: 65-66) han sugerido que las políticas públicas dirigidas a enfrentar los problemas derivados del envejecimiento generacional en Uruguay, país con el mayor porcentaje de población mayor de 60 años en América Latina, deben buscar la equidad intergeneracional:

Probablemente está claro que los actuales "adultos mayores" no son prioritarios en la agenda social, sin embargo una vez profundizado el tema son varias las cosas por hacer sin perjuicio de la atención a otros grupos etáreos más afectados por la inequidad social en la actual coyuntura. 
En este tenor, la Cepal (2000) advierte que en América Latina la condición socioeconómica de los adultos mayores no es más devastadora que otros sectores de la población, los niños en particular; y que no es la pobreza en sí la causa de su estado de vulnerabilidad, sino que su bienestar se ha visto deteriorado por la falta de sistemas de seguridad social de amplia cobertura al momento del retiro, fragilidad de los sistemas de salud, falta de una política de vivienda y de mecanismos de integración social e intergeneracional. De lo anterior se desprende la necesidad de investigaciones y propuestas integradas al desarrollo humano sostenible, cuyo objetivo sustancial sea elevar y mejorar la calidad de vida de todos los miembros de la sociedad, particularmente los pobres o vulnerables (Viveros, 200I). Tal como lo ha expresado Dick Jaspers (2007a: 38): "Los gobiernos deberían analizar las políticas que vienen implementando y reforzar aquellas dirigidas a establecer una sociedad que incluya a todos, en lugar de circunscribir las actividades hacia las personas mayores como asuntos alejados del devenir de una sociedad".

No faltan, pues, propuestas integrales de atención necesarias para enfrentar los problemas que surgen del cambio demográfico. Lo que prevalece es un vacío legislativo y una disposición plena que las considere. Viveros (200I: 17) encuentra que por lo menos son tres los factores que dificultan la acción social al respecto: 1) La incapacidad de los países para identificar la magnitud de los problemas; 2) Una cierta "pereza pública" para asumir las recomendaciones de organismos internacionales; 3) El déficit y la incapacidad de recursos públicos y privados para hacer frente al nuevo cuadro de demandas originadas por el envejecimiento. Yo añado una más: la opacidad de las políticas públicas que se dirigen específicamente a un sector de la población y sus problemas en lo particular, que no hacen más que atomizar la búsqueda y defensa de derechos propios de otros sectores y grupos de edad.

Una política pública integral hacia los adultos mayores debe comenzar por garantizar la seguridad social y el acceso a la justicia de toda la población, y no solo de aquella considerada más vulnerable, como es el caso de los ancianos en México. Debe contemplar necesariamente problemáticas a las que se enfrenta la población en general, puesto que de ello dependerá que la población más joven supere o no las condiciones de miseria y desigualdad que hoy padecen los adultos. Esta perspectiva no menosprecia las políticas públicas que permiten la identificación de áreas de intervención en particular, a partir de las cuales se atienden necesidades propias de la población mayor, pero insiste en la necesidad de construir políticas públicas no privativas de otros sectores que contemplen la atención de la población más joven, de manera tal que se garantice una vejez autosuficiente a partir de políticas de prevención y no solo de asistencia. Lo anterior se vuelve necesario puesto que 
el envejecimiento generacional se impone sin que se hayan resuelto problemas tan básicos como la desigualdad social, la pobreza, la falta de fuentes de empleo decente, servicios eficientes de salud, así como la inseguridad y las barreras para un acceso pleno a la justicia. De ahí que a la par de pensar en programas de acción dirigidos a la población adulta, escribe Roberto Ham (2010: 59-75), es importante pensar también en propuestas de una mejor sociedad frente al envejecimiento, orientadas a mejorar las relaciones familiares, las estructuras económicas y los pactos sociales, considerando a las generaciones más jóvenes que a futuro llevarán la carga mayor. Las propuestas también deben apelar no solo al pensamiento especializado, sino al sentido común que reconsidere, por ejemplo, sobre derechos adquiridos que se forzaron a ser legales, pero siempre han sido injustos; manejo político para que se acepte renunciar a prebendas; generosidad, búsqueda del bien común y miradas a largo plazo por el futuro bienestar del país.

\section{Comentarios finales}

En síntesis, como primera conclusión, he pretendido mostrar la inviabilidad del paradigma de políticas públicas dirigidas exclusivamente a cubrir las necesidades de las personas mayores desde una perspectiva proteccionista y asistencialista, puesto que este modelo de intervención evita considerar problemáticas de fondo que anteceden al envejecimiento de las personas; son medio y objeto de corrupción y despojo; y su impacto es prácticamente nulo en relación con los objetivos que se pretenden alcanzar. Debido a lo anterior, se están encauzando recursos a la población adulta mayor de manera tal que la población que se aproxima a esa etapa pareciera esperar a envejecer para recibir los beneficios de las políticas asistencialistas, en lugar de procurar el acceso a una mayor plenitud en la calidad de vida y los derechos desde que las personas son más jóvenes. En vez de pensar en derechos particulares, debe buscarse mejorar el acceso a la salud, el empleo, la justicia y la equidad de toda la población.

La segunda conclusión es que se debe ir un paso adelante y superar un asistencialismo limitado a la distribución de recursos materiales y económicos como forma de ayuda a las personas y grupos sociales considerados de facto vulnerables y, en vez de ello, establecer una política pública de justicia social, dejando así de tratarlos como sujetos de asistencia e integrarlos como sujetos de derechos. La extrema atomización de derechos dirigidos a sectores sociales particulares olvida considerar la relación estrecha con las necesidades de otros sectores. ¿No es más incluyente y necesario 
pensar en mejorar la calidad de vida de las personas en general, en lugar de pensar en mejorar las condiciones de vida de un sector en particular por ser considerarlos como "los más vulnerables"? ¿No es más incluyente demandar el respeto pleno a los derechos de las personas, y no pensar en derechos particulares para los ancianos por una parte y para los no ancianos por otra? Se ha considerado como población vulnerable a ciertos sectores de la sociedad: niños, indígenas, mujeres, madres solteras $y$ adultos mayores, $y$ de ese modo se ha legislado en aras de beneficiarlos a partir de programas de asistencia. Al hacerlo, se han fragmentado los derechos de las personas y de amplios sectores, protegiendo a unos e ignorado los derechos de otros.

Finalmente, no puede negarse que la población adulta padece actos discriminatorios, y que se requieren políticas públicas orientadas a mejorar su calidad de vida. No obstante, y como tercera conclusión, sugiero que las condiciones de desigualdad y las distintas formas de segregación social que padecen los adultos mayores en México no se deben a la discriminación en sí misma, sino a las condiciones de miseria en que vive gran parte de la población; a la inseguridad social y económica; a las condiciones de vida azarosas e inestables; a la violencia que se genera ante la necesidad de superar circunstancias adversas; a la falta de reconocimiento de derechos humanos elementales, y a la disfuncionalidad del Estado e incompetencia de su burocracia para atender las necesidades de la población en general. Finalmente, algunos académicos hablan más de discriminación por estereotipos y prejuicios hacia la vejez que los propios adultos mayores. Posiblemente el sesgo esté bien arraigado a sus propias experiencias de vida, que les generan ciertos sentimientos al enfrentarse a procesos inherentes a su edad, al encarar dificultades de sus ambientes de trabajo y a consideraciones de su intelectualidad.

\section{Bibliografía}

Alba, Víctor (1992), "Historia social de la vejez", Lahertes, Barcelona. Alonso, María del Pilar (2012), Políticas públicas de pensiones en México orientadas a la población sin capacidad de ahorro, México, Instituto Nacional de Administración Púbica.

Axelrod, Seymour y Carl Eisdorfer (196I), "Attitudes toward old people: an empirical analysis of the stimulus group validity of the Tuckman-Lorge questionnaire" en Journal of Gerontology, 16, pp. 75-80. 
Becca, Levy y Mahzarin Banaji (2004), "Implicit ageism”, en Todd D. Nelson (comp). Ageism: stereotyping and prejudice against older persons, Massachusetts, The Mit Press.

Brewer, Marilynn, Valerie Dull y Layton Lui (198I), "Perceptions of the elderly: Stereotypes as prototypes", Journal of Personality and Social Psychology, 4I(4), pp. 656-670.

Brubaker, Timothy y Edward Powers (1976), "The stereotype of old: A review and alternative approach", Journal of Gerontology, 3I(4), pp. 44I-447.

Bucio, Ricardo y Alejandro Lucas (201I), "Presentación”, en Encuesta Nacional sobre Discriminación en México 2010. Resultados sobre personas adultas mayores, México, INAPAM-CONAPRED.

Butler, Robert (1969), "Age-ism: Another form of bigotry", Gerontologist, 9(4), pp. 243-246.

Caro, Elizabeth (2003), "Nuevas políticas para los adultos mayores: el caso del Distrito Federal. De la Asistencia a la participación social”, Revista de Administración Pública, 109, pp. 75-86.

CEPAl (2000), Panorama Social de América Latina, 1999-2000, Naciones Unidas, Santiago de Chile.

Cepal (2007), Programa Social de América Latina, Naciones Unidas, Santiago de Chile.

CEPAl (20IO), Estudio económico de América Latina y el Caribe. Modalidades de inserción externa y desafios de política macroeconómica en una economía mundial turbulenta, Naciones Unidas, Santiago de Chile.

Chasteen Alison, Norbert Schwarz y Denise Park (2002), "The Activation of Aging Stereotypes in Younger and Older Adults", Journal of Gerontology, 57(6), pp. 540-547.

Cohen, Elias (200I), "The Complex Nature of Ageism What Is It? Who Does It? Who Perceives It?", The Gerontologist, 4I(5), pp. 576-577.

Consejo Nacional de Población (2006); Proyecciones de la población de México 2005-2050, CONAPO, México.

conapo (20II), Diagnóstico socio-demográfico del envejecimiento en México, México, Conapo (serie Documentos técnicos).

Cook, Fay (1992), "Ageism: Rhetoric and Reality", The Gerontologist, 32(3), pp. 292-293.

Davidovic, Mladen, Zorana Djordjevic, Predrag Erceg, Nebojsa Despotovic y Dragoslav P. Milosevic (2007), "Ageism: does it exist among children?”, Scientific World Journal, 27(7), pp. II34-II39. 
Díaz, Aída (200I), "Estudios de Población y enfoques de Gerontología Social en México", Papeles de Población, I7(70), octubre-diciembre, pp. 49-79. Dulcey, Elisa y Rubén Ardilla (1976), "Actitudes hacia los ancianos", Revista Latinoamericana de Psicología, 8(I), pp. 57-67.

Fernández, Antoni (1997), "Las políticas públicas", en Manual de ciencia politica, Miquel Caminal (ed), Madrid, Tecnos.

Galtung, Johan (1994), Human rights in another key, Polity Press, Cambridge. Garay Sagrario y Verónica Montes de Oca (20II), "La vejez en México: una mirada general sobre la situación socioeconómica y familiar de los hombres y mujeres adultos mayores", Perspectivas Sociales/Social Perspectives, vol. I3, núm. I, pp. I43-165.

Geertz, Clifford (1997), La interpretación de las culturas, Barcelona, Gedisa.

González de la Rocha, Mercedes (2006), "Introducción”, en Mercedes González de la Rocha (coord). Procesos domésticos y vulnerabilidad. Perspectivas antropológicas de los hogares con Oportunidades, México, CIESAS.

Ham, Roberto (1999), "El envejecimiento en México: de los conceptos a las necesidades", Papeles de Población, 5(19), pp. 7-2I.

Ham, Roberto (2010), "Envejecimiento demográfico", en Brigida García y Manuel Odorica (coords). Los grandes problemas de México, I Población, México, El Colegio de México.

Hausdorff Jeffrey, Levy Becca y Jeanne Wei (1999), “The power of ageism on physical function of older persons: Reversibility of age-related gait changes", Journal of the American Geriatrics Society, 47(II), pp. I346-I349.

Henríquez, José, Julia Johannsen y Natasha Morales (2015), "La coordinación entre actores en la implementación de programas sociales: dos estudios de caso", en Alejandro Bonvecchi, Julia Johannsen y Carlos Scartascini (eds.), ¿Quiénes deciden la politica social? Economía politica de programas sociales en América Latina, Nueva York, Banco Interamericano de Desarrollo.

Huenchuan, Sandra (2003), "Políticas de vejez en américa latina: una propuesta para su análisis", Simposio Viejos y Viejas Participación, Ciudadania e Inclusión Social, $5^{\circ}$ Congreso Internacional de Americanistas, Santiago de Chile.

Huenchuan, Sandra (2004), "Marco legal y de políticas a favor de las personas mayores en América Latina”, serie Población y desarrollo, 5I, Santiago de Chile, onu. 
Huenchuan, Sandra (2009), Envejecimiento, derechos humanos y politicas públicas, Santiago de Chile, onu.

Huenchuan, Sandra y José Guzmán (2006), “Seguridad Económica y Pobreza en la Vejez: Tensiones, Expresiones y Desafíos para Políticas”, Reunión de Expertos sobre Población y Pobreza en América Latina y el Caribe, Santiago de Chile, Cepal, Celade.

Huenchuan, Sandra, Daniela González, Mariana Paredes y José Guzmán (2007), Protección y participación en la vejez: escenarios futuros y politicas públicas para enfrentar el envejecimiento en Chile, Santiago de Chile, onu.

Hummert, Mary (1990), "Multiple Stereotypes of Elderly andlbung Adults: A Comparison of Structure and Evaluations", Psichology and Aging, 5(2), pp. I82-193.

inapam-Conapred (20II), Encuesta Nacional sobre Discriminación en México 20I0, Resultados sobre personas adultas mayores, México, INAPAM-CONAPRED.

INEGI (2005), Los adultos mayores en México. Perfil sociodemográfico a inicio del siglo XXI, México, INEGI.

Jaspers, Dick (2007a), Envejecimiento y desarrollo en una sociedad para todas las edades, Santiago de Chile, CePal-CELAde.

Jaspers, Dick (2007b), "Introducción”, en Sandra Huenchuan y Daniela González, Protección y participación en la vejez: escenarios futuros y politicas públicas para enfrentar el envejecimiento en Chile, Santiago de Chile, Documento de Proyecto, ONU.

Jiménez, Araceli (20I2), "Violencia en la vejez: el caso de las abuelas que cuidan a nietos y nietas en una localidad rural en el estado de Hidalgo", $E l$ Cotidiano, I74, pp. 19-32.

Lasagni, Viviana (2012), Estereotipos hacia la Vejez en Adultos Mayores y en Estudiantes Universitarios en diez paises de América Latina, Santiago de Chile, Red Latinoamericana de Gerontología.

Lovell, Marge (2006), "Caring for the elderly: Changing perceptions and attitudes" Journal Vascular Surgery, 24(I), pp. 22-26.

Lozano, Arturo (2009), "La gerontocracia y la gerontofobia", Revista Facultad de Medicina, unAm, 52(6), pp. $265-267$.

Lyonnais, Groupe (1965), "La vejez problema de hoy”, Razón y fe, Madrid. 
Marín, Pedro (2006), "Lineamientos para la reformulación de la política pública de salud para personas mayores", Temas de la agenda pública, I(5), Pontificia Universidad Católica de Chile.

Matheson Devora, Caroline Collins y Valery Kuehne (2000), "Older adults' multiple stereotypes of young adults, The International Journal of Aging and Human Development, 5I(4), pp. 245-257.

Meny, Ives y Jean-Claude Thoenig (1992), Las politicas públicas, Barcelona, Ariel.

Montes de Oca, Verónica (1999), "Diferencias de género en el sistema de apoyo a la población envejecida en México", Papeles de Población, 5(19), pp. 149-172.

Montes de Oca, Verónica (2000), "Experiencia institucional y situación social de los ancianos en la Ciudad de México”, en Rolando Cordera y Alicia Ziccardi (Coords). Las políticas sociales en México al fin del milenio. Descentralización, diseño y gestión, México, unAm-Porrúa.

Montes de Oca, Verónica (2013), Envejecimiento en América Latina y el Caribe. Enfoques interdisciplinarios en investigación y docencia de la Red Latinoamericana de Investigación en Envejecimiento, México, unam.

Montes de Oca, Verónica (20I3b), "La discriminación hacia la vejez en la ciudad de México: contrastes sociopolíticos y jurídicos a nivel nacional y local", Perspectivas Sociales/Social Perspectives, I5(I), pp. 47-80.

Moragas, Ricardo (1998), Gerontología social: Envejecimiento y Calidad de vida, Barcelona, Herder.

Moreno, Ángel (20II), "Viejismo (ageism). Percepciones de la población acerca de la tercera edad: estereotipos, actitudes e implicaciones sociales", Poiésis, 19, pp. I-IO.

Muñoz, Roxana (20II), "Las políticas públicas de la vejez en México 20ıо" en Iztapalapa, Revista de Ciencias Sociales y Humanidades, 32(7I), pp. 35-60.

Ordorica, Manuel (2010), "Las proyecciones de la población hasta la mitad del siglo XXI", en Brigida García y Manuel Ordorica (coords). Los grandes problemas de México, Población I, México, El Colegio de México.

Palmore, Erdman (1990), "Ageism: Negative and positive, Springer, Series on Adulthood and Aging, Nueva York, Springer Pub. Co.

Paz, Jorge (20I0), "Envejecimiento y empleo en América Latina y el Caribe", Documento de trabajo Núm. 56, Suiza, Organización Internacional del Trabajo. 
Piña, Marcelo (2006), "Trabajo social gerontológico: investigando y construyendo espacios de integración social para las personas mayores", Rumbos TS, I(I), I3-30.

Polo, María y Mari Martínez (200I), "Visión histórica del concepto de vejez en las sociedades antiguas", Revista de los cuidados, 5(IO), pp. I5-20.

Ramírez, Nohora y Alba Cruz (2010), "Escenario conceptual para la formulación de política pública en vejez y envejecimiento", Tend. Retos, I5, pp. 83-94.

Razo, Angélica (20I4), "La política pública de la vejez en México: de la asistencia púbica al enfoque de derechos", Revista Conamed, 19(2), pp. 78-85.

Regil, Miriam de (20I4), Más de un millón de ancianos padecen violencia en México, El Financiero, 26-05-I4, en <http://www.elfinanciero.com. $\mathrm{mx} /$ sociedad/mas-de-un-millon-de-ancianos-padecen-violencia-enmexico.html> [consulta: 10/07/2015].

Reyes, Laureano, Ana Berónica Palacios Gámaz, Socorro Fonseca Córdoba y Susana Villasana Benítez (2013), “ La gerontocracia y el consejo de ancianos", Peninsula, 8(I), pp. 7-24.

Ribeiro, Manuel y Sandra Mancinas (2010), Textos y Contextos del Envejecimiento en México, México, Plaza y Valdés.

Robles, Leticia, Felipe Vázquez, Laureano Reyes e Imelda Orozco (2006), Miradas sobre la vejez. Un enfoque antropológico, Tijuana, El Colegio de la Frontera Norte / Plaza y Valdés.

Rodríguez, Sandalio (1989), La vejez, historia y actualidad, Salamanca, Universidad de Salamanca.

Rojas, Abigail (20II), "Pensiones en la población femenina de edades avanzadas en México", XI Reunión Nacional de Investigación Demográfica en México, Ciudad de Aguascalientes, 30 de mayo al I de junio.

Rosas, Denhi (2013), "México no ha tenido políticas públicas para adultos mayores", Boletín UNAM-DGCS-685, Ciudad Universitaria, en <http:// www.dgcs.unam.mx/boletin/bdboletin/2013_685.html>, [consulta: 03/03/20I5]

Ruiz, Elisa, Deisy Sánchez y Pablo Sanabria (2013), Envejecimiento y vejez en Colombia. Aportes para una politica pública, Bogotá, ENDS 1990-2010 (serie Estudios a Profundidad).

SAGARPa (20I4), Estudio sobre el envejecimiento de la población rural en México , México, sagarpa. 
Salazar, Héctor (2015), "Prólogo", en Alejandro Bonvecchi, Julia Johannsen y Carlos Scartascini (eds.), ¿Quiénes deciden la política social? Economía politica de programas sociales en América Latina, Nueva York, Banco Interamericano de Desarrollo.

Sánchez, Concepción (2004), Estereotipos negativos hacia la vejez y su relación con variables sociodemográficas, psicosociales y psicológicas, tesis doctoral inédita, Universidad de Málaga.

Scartascini, Carlos (2015), "La economía política de las políticas públicas", en: Alejandro Bonvecchi, Julia Johannsen y Carlos Scartascini (eds). ¿Quiénes deciden la politica social? Economía politica de programas sociales en América Latina, Nueva York, Banco Interamericano de Desarrollo.

Schonfield, David (1982), "Who is stereotyping whom and why?", The Gerontologist, 22(3), 267-272.

Secretaría de Desarrollo Social (2013), Diagnostico del programa pensión para adultos mayores, México, SEDESOL.

Tourliere, Mathieu (2015), "Desnuda la ASF fraude masivo en Cruzada contra el Hambre", Proceso, 19-02-I5, en <http://www.proceso. com.mx/396327/2015/02/19/la-cruzada-nacional-contra-el-hambre-un-fraude-masivo>, [consulta: 20/05/2015],

Vargas, Emma, Cecilia Velázquez, Liliana Galicia, Enrique Villareal y Lidia Martínez (20II), "Tipo de violencia familiar que percibe el adulto mayor", Revista de Enfermería del Instituto Mexicano del Seguro Social, I9(2), pp. 63-69.

Viveros, Alberto (200I), Envejecimiento y vejez en América Latina y el Caribe: políticas públicas y las acciones de la sociedad, Santiago de Chile, ONU.

Weigert, Kathleen (1999), "Structural Violence", en Lester A. Kurtz (ed). Encyclopedia of Violence, Peace and Conflict, vol. 3, Academic Press, San Diego, pp. 43I-440.

Wong, Rebeca (2006), "Envejecimiento en áreas urbanas marginadas de México: condiciones mixtas de privilegio y desventaja", en Nelly Salgado y Rebeca Wong (eds.). Envejecimiento, pobreza y salud en población urbana. Un estudio en cuatro ciudades de México, México, Instituto Nacional de Salud Pública.

Zizek, Slavoj (2009), Sobre la Violencia: Seis Reflexiones Marginales, Buenos Aires, Paidós. 
Resumen CURRICUlar

Doctor en Ciencias Antropológicas por la Universidad Autónoma Metropolitana. Posdoctorado en el Centro de Investigaciones y Estudios Superiores en Antropología Social (CIESAS-golfo). Diplomado en Escritura de textos científicos y literarios, Formación pedagógica, Análisis de la cultura, Filosofía de la educación en América Latina, Teoría e historia de las religiones, Relaciones de género, Historia y hermenéutica del mito. Últimos artículos publicados: "Trascendencia de las creencias religiosas entre adultos mayores en grupos de Alcohólicos Anónimos", Nómadas. Revista crítica de ciencias sociales y jurídicas, vol. 45, núm. I, 2015, pp. 133I49; "Rituales de degradación en la práctica docente", Revista Educación y Desarrollo, Universidad de Guadalajara, núm. 34, 2015, pp. 5I-62; "Encrucijadas de la fe. El impacto de la migración y la conversión religiosa en los procesos de construcción de comunidades interculturales", María Luisa Quintero Soto y Elisa Bertha Velázquez (coords.), Realidades Interculturales: miradas hacia el género y la educación, México: Castellanos editores, 2015, pp. 153-176.

Citar como: Óscar Osorio Pérez (2016), "Envejecimiento poblacional: discriminación y políticas públicas integrales", Iztapalapa. Revista de Ciencias Sociales y Humanidades, núm. 8I, año 37, julio-diciembre de 20I6, ISSN: 20079176; pp. 141-172. Disponible en < http://revistaiztapalapa.izt.uam.mx/ index.php/izt/issue/archive > 